\title{
Evaluation of the Efficacy of Methylprednisolone Pulse Therapy in Treatment of Covid-19 Adult Patients with Severe Respiratory Failure: Randomized, Clinical Trial
}

\section{Ramin Hamidi Farahani}

Aja University of Medical Sciences

\section{Reza Mosaed}

Aja University of Medical Sciences

Amir Nezami-Asl

Aja University of Medical Sciences

Mohsen chamanara

Aja University of Medical Sciences

\section{Saeed Soleiman-Meigooni}

Aja University of Medical Sciences

\section{Shahab Kalantar}

Aja University of Medical Sciences

Mojtaba Yousefi zoshk

Aja University of Medical Sciences

\section{Ebad Shiri Malekabad}

Aja University of Medical Sciences

Ebrahim Hazrati ( $\nabla$ Dr.hazrati.e@gmail.com )

Aja University of Medical Sciences

\section{Research}

Keywords: Coronavirus disease 2019 (COVID-19), methylprednisolone pulse, corticosteroids, clinical trials

Posted Date: September 9th, 2020

DOl: https://doi.org/10.21203/rs.3.rs-66909/v1

License: (c) (1) This work is licensed under a Creative Commons Attribution 4.0 International License.

Read Full License 


\section{Abstract}

Background: Covid-19 is now global concern and widely spread to the world due to high mortality among the nations we tried to evaluate the efficacy of methylprednisolone pulse in COVID-19 patients with severe respiratory failure.

Methods: This study was phase2, double-blind, randomized, clinical trial in adults with COVID-19 (aged $\geq 18$ years old) admitted to the intensive care unit (ICU) of *. Patients with intermediate or severe COVID19 with $\mathrm{PaO} 2 / \mathrm{FiO} 2$ less than 300 and progressive disease unresponsive to standard treatments admitted to ICU. Patients were randomly allocated in either control or investigation group. The control group received recommended regimen for COVID-19. The investigation group received the recommended regimen plus Methylprednisolone (1000 mg/day for three days) and oral prednisolone $1 \mathrm{mg} / \mathrm{kg}$ with tapering of dose within ten days.

Results: A total of 29 ICU patients with intermediate or severe COVID-19 pneumonia recruited in this study. Fourteen patients (4 female, ten male) allocated in the investigation group, and 15 patients (6 female, nine male) assigned to the control group. The participant's average age was $64.03 \pm 13.545$ (case: $61.07 \pm 12.83$, control: $66.80 \pm 14.03)$. The patients with methylprednisolone pulse had significantly higher systolic $(P=0.018)$ and diastolic $(P=0.001)$ blood pressure, meanwhile, the Glasgow coma scale $(G C S)$ of methylprednisolone group was considerably $(\mathrm{P}<0.001)$ higher, and by the improvement in SpO2 of methylprednisolone group none of these patients needed mechanical ventilation.

Conclusion: This study demonstrated methylprednisolone pulse in COVID-19 severe respiratory failure dramatically improves the clinical condition of patients including, GCS, and SpO2 of patients.

\section{Clinical Trial Registration Number: IRCT20200406046963N1}

\section{Introduction}

In December 2019, coronavirus disease 2019 (COVID-19) was first reported in Wuhan, the capital of Hubei, China, which rapidly spread to the rest of the world [1]. The world health organization (WHO) declared the COVID-19 outbreak as a Public Health Emergency of International Concern (PHEIC). By June 14,2020 , the total number of patients reached approximately 8 million cases and half a million death across the world[2]. The clinical manifestation of COVID-19 ranges from asymptomatic, mild pneumonia to acute serious respiratory failure, septic shock, and multiple organ dysfunction syndromes (MODS) [3, 4]. Patients with intermediate or severe symptoms and respiratory failure would need to be admitted to ICU[5]. COVID-19 spreads through respiratory droplets or direct contact. It appears to have higher infectivity but a lower case fatality rate compared to severe acute respiratory syndrome (SARS) and Middle Eastern respiratory syndromes (MERS)[6, 7].

Studies showed pathological characteristics of COVID-19 pneumonia greatly resemble the pathologic features of SARS and MERS, representing a bilateral diffuse alveolar damage with cellular fibromyxoid 
exudates, and the desquamation of pneumocytes and hyaline membrane formation, indicating acute severe respiratory failure [8]. Moreover, microvascular thrombosis and hemorrhage with micro-angiopathy in small vessels and capillaries of lungs are reported in COVID-19 patients, which significantly contributed to the death[9]. Although corticosteroid treatment is not routinely recommended to be administered for COVID-19 patients, as $Z$ he $X u$ [8] proposed, due to pulmonary edema and hyaline formation use of corticosteroids might prevent severe respiratory failure development in severe patients.

Patient management's core essence in COVID-19 is antiviral and supportive therapy, including fluid management, oxygen therapy, and mechanical ventilation[10]. As cytokine storm is relatively common in severe cases and often leads to exacerbation[11], several anti-inflammation treatments are proposed, including non-steroidal anti-inflammatory drugs, and glucocorticoids, chloroquine/hydroxychloroquine, immunosuppressant, inflammatory cytokines antagonists[12]. Concerning the widespread use of corticosteroids as a therapy for ARDS [13]and proof of efficacy to treat SARS, which could significantly decrease the mortality of severe SARS patients, we aimed to evaluate the efficacy of methylprednisolone pulse on intermediate, and severe COVID-19 patients admitted to Intensive Care Unit (ICU).

\section{Material And Method}

\section{Study design:}

From April 2020 to May 2020, we performed phase2, double-blind, randomized, clinical trial to assess the effectiveness and safety of methylprednisolone pulse in adults with COVID-19 (aged $\geq 18$ years old) admitted to ICU of * Hospital of *.. Study protocols were reviewed by the ethics committee of AJA University (application number: IR.AJAUMS.REC.1399.008), and the trial was registered at www.irct.ir (Reference number: IRCT20200406046963N1). All patients provided written informed consent.

\section{Patients:}

We enrolled patients 18 years of age or older with moderate to severe Covid-19 infection who admitted to $\mathrm{ICU}$ with $\mathrm{PaO} 2 / \mathrm{FiO} 2$ less than 300 and progressive disease not responding to recommended treatment with the prediction of need for intubation within next 24 hours, patients with uncontrolled diabetes mellitus, Active GI bleeding, history of corticosteroid hypersensitivity, severe electrolyte imbalances, Procalcitonin more than 0.5 , active bacterial, viral (HIV, Hepatitis), and fungal infection were excluded from the study. Laboratory confirmation of COVID-19 infection was made using the reverse-transcription polymerase chain reaction (RT-PCR).

Patients were randomly (with random allocation software) assigned to either investigation group: receiving recommended regimen (Kaletra [lopinavir/ritonavir] daily, Hydroxychloroquine $400 \mathrm{mg}$ daily, Azithromycin 500 mg daily) for COVID-19 plus Methylprednisolone (1000 mg/day for three days), and oral prednisolone $1 \mathrm{mg} / \mathrm{kg}$ with tapering of dose within ten days or Control group: receiving recommended regimen for COVID-19. 


\section{Outcomes:}

The primary objective of this study was to investigate the efficacy of methylprednisolone pulse on mortality rate, blood 02 saturation, and need for further oxygen therapy. Sequential Organ Failure Assessment (SOFA) Score changes were assessed before and during the study. Moreover radiographic and laboratory parameters including Creatine phosphokinase (CPK), lactate dehydrogenase (LDH), and serum creatinine $(\mathrm{SrCr})$, Total and Direct bilirubin were studied.

\section{Statistics:}

Data analysis was performed using IBM SPSS 16 for windows (Chicago, IL, USA). A P-value $<0.05$ was considered statistically significant. Continues variables were reported as mean, standard deviation, and then were compared by student's T-test.

\section{Results}

A total of 29 ICU patients with intermediate or severe COVID-19 pneumonia recruited in this study. Patients randomly assigned to the investigation and control group, 14 patients ( 4 female, ten male) allocated in the investigation group, and 15 patients ( 6 female, nine male) assigned to the control group. The participant's average age was $64.03 \pm 13.545$ (case: $61.07 \pm 12.83$, control: $66.80 \pm 14.03$ ).

Oxygen therapy, antiviral therapy (Kaletra [lopinavir/ritonavir]), prevention of bacterial infection, and nutritional support were administered for all 29 patients. While the case group received methylprednisolone pulse with the dosage of $1000 \mathrm{mg} /$ day for three days via intravenous injection. There was no mortality among the patients receiving the methylprednisolone treatment, but the mortality was high in patients without methylprednisolone therapy.

The mean systolic blood pressure (SBP) at rest was significantly (P-Value:0.018) higher among patients with methylprednisolone pulse therapy compared to the control group on admission day and remained higher on following ten days. The daily mean SBP of the two groups shown in Fig. 1. Patients with methylprednisolone therapy had a mean SBP of $115 \mathrm{mmHg}$ on admission day, which raised steadily to $125 \mathrm{mmHg}$ on the tenth day. Meanwhile, patients without methylprednisolone had a mean SBP of 105 on admission day, which fluctuated on the following ten days and reached to $120 \mathrm{mmHg}$ on the sixth day but finally returned to $105 \mathrm{mmHg}$ on the tenth day.

The mean diastolic blood pressure (DBP) at rest was significantly (P-Value: 0.001) higher among patients with methylprednisolone pulse therapy on admission day, mean DBP of patients with methylprednisolone stayed constant on $80 \mathrm{mmHg}$ with a little fluctuation but the mean DBP of patients without methylprednisolone raised from $70 \mathrm{mmHg}$ to $80 \mathrm{mmHg}$ with intense fluctuation in following ten days. The daily mean DBP of case and control groups shown in Fig. 2.

The mean FiO2 administered for patients with or without methylprednisolone pulse therapy on admission day was similar, due to low Sp02, all the patients with or without methylprednisolone therapy received 
oxygen therapy. The daily mean FiO2 administered and SpO2 of patients in case and control group are shown in Fig. 3 and Fig. 4, respectively. As shown in Fig. 3, FiO2 needed for patients with methylprednisolone therapy dropped steadily from approximately $90-50 \%$, and the need for Positive endexpiratory pressure (PEEP) declined from $10 \mathrm{cmH} 2 \mathrm{O}$ to $5 \mathrm{cmH} 2 \mathrm{O}$ as shown in Fig. 5 . In addition to improvement of respiratory outcome in methylprednisolone group, Glasgow Coma Scale (GCS) of methylprednisolone group significantly $(P-V a l u e<0.001)$ improved since the administration of methylprednisolone as presented in Fig. 6 (mean GCS of 15 in methylprednisolone group versus GCS of lower 10 in the control group)

The laboratory parameters, including CPK, LDH, and SrCr, were significantly (CPK, P-Value $=<0.001, \mathrm{LDH}$, $\mathrm{P}$-Value $=0.003, \mathrm{SrCr}, \mathrm{P}$-Value $=<0.001$ ) different among patients with and without methylprednisolone group. Both mean CPK and mean LDH decreased through 10 days follow up, but drop and fluctuation of these measures were more intense in the control group (Fig. 7, Fig. 8). Moreover, there was no significant difference in trends of mean Total and Direct bilirubin among the case and control groups.

Assessment of patient's Chest CT-scan pretreatment (Fig. 9) and 3 days post treatment (Fig. 10) in ICU revealed the significant clearance of Chest scan from patchy ground-glass lesions without any trace of fibrosis in patients receiving the methylprednisolone while there were no evidence of recovery in chest CTscan of the control group.

\section{Discussion}

This trial found that methylprednisolone pulse significantly improved the general condition of patients with intermediate and severe patients of COVID-19 who admitted to ICU; GCS, SpO2 of patients dramatically improved after the administration of methylprednisolone which decreased the further need for mechanical ventilation in methylprednisolone group. GCS of patients on methylprednisolone therapy was significantly higher than patients not receiving methylprednisolone (GCS of 15 in the methylprednisolone group versus GCSs of lower 10 in the control group among ten-day follow up-Figure 6). SpO2 of methylprednisolone group was also significantly higher compared to the control group, which decreased the demand for higher FiO2 or PEEP in patients receiving methylprednisolone therapy, as shown in Fig. 3, Fig. 5 respectively).

We recognized that symptoms of patients aggravate during the 7-10 after the disease onset and progress to severe respiratory failure. In this phase patient's SpO2 dropped rapidly with an increase in erythrocyte sedimentation rate (ESR) and C-reactive protein (CRP) levels. Meanwhile, radiographic chest progression was obvious, but these patients were not as toxic as other cause ARDSs, and they were fully conscious and didn't fulfill any Systemic Inflammatory Response Syndrome (SIRS) criteria. Despite of treatment continuation with recommended protocol in this phase, patients needed more respiratory support such as Noninvasive positive-pressure ventilation (NIPPV), and intubation in severe cases. Because of COVID-19 different pathology in comparison with ARDS, using routine ARDS treatment protocols were unsuccessful. 
Our patient's chest radiographic presentation was similar to interstitial lupus pneumonitis, which is a rare and also highly lethal manifestation of systemic lupus erythematosus[14]. We recognized diffuse fibrotic changes and parenchymal consolidation in our patient's chest radiographs which is similar to radiographic features of lupus pneumonitis $[15,16]$ regarding the immunological origin of lupus pneumonitis we hypothesized that immunological processes might be involved in the pathogenesis of COVID-19 lung injury, and immunosuppressive treatment might improve the patient's condition. As clearance of chest CT-scan of patients receiving methylprednisolone from patchy ground-glass in current study confirmed this notion.

Mc Gonagle et al. illustrated that lung pathology seen in patients with COVID-19 shows marked alveolar and interstitial inflammation[17] It is currently believed that lung injury in COVID-19 not only associates with direct viral injury but also COVID-19 triggers immune response which leads to activation of immune cells and cytokine storm[18]. Cytokine storm extensively damages vascular endothelium and alveolar epithelium which increases vascular permeability leading to pulmonary edema and hyaline formation[19, 20], similar to our findings previous studies also reported aggravation of symptoms during 5-7 days after the disease onset[21], therefore it is important to control the inflammatory response and cytokine storm in this phase, however, by the systematic review, Nicola Veronese concluded that the routine use of corticosteroids in COVID-19 couldn't be encouraged, but methylprednisolone therapy could lower mortality rate in more severe forms of this condition, such as in severe respiratory failure.[22] So with the assumption of an immune-mediated incident, in moderate to severe patients, we started using high dose steroid pulse therapy with $1000 \mathrm{mg}$ methylprednisolone sodium succinate for three days, which continued with oral prednisolone $(1 \mathrm{mg} / \mathrm{kg})$, while tapering the dose within seven days. The general condition of all patients receiving methylprednisolone pulse was significantly improved as none of them didn't need for mechanical ventilation, and they were dischargeable from ICU after ten days of receiving corticosteroid. Similar to our findings, Yin Wang also previously reported intravenous administration of methylprednisolone with a dosage of $1-2 \mathrm{mg} / \mathrm{kg} / \mathrm{d}$ for $5-7$ days in COVID-19 patients with severe pneumonia was associated with faster improvement of clinical symptoms, SpO2, and higher absorption degree in chest CT[23]. On June 16, RECOVERY (Randomized Evaluation of COVid-19 therapy) trial also established dexamethasone reduces deaths by one third in hospitalized patients with the severe respiratory complication of COVID-19[24].

However, primary studies could not suggest the corticosteroid use for COVID-19 patients; new studies, including our findings, suggests corticosteroid administration on intermediate or severe COVID-19 patients would improve the clinical outcome of patients.

\section{Limitation:}

Due to the limited number of patients in our trial, further investigations will be needed to explore the efficacy and possible complications of methylprednisolone pulse in the treatment of intermediate and severe COVID-19 patients. 


\section{Conclusion}

Our study showed methylprednisolone pulse in COVID-19 patients with severe symptoms and severe respiratory failure would dramatically improve the clinical condition, GCS and SpO2 of patients.

\section{Declarations}

Ethics approval and consent to participate: The protocols were reviewed by the ethics committee of AJA University (application number: IR.AJAUMS.REC.1399.008), All patients provided written informed consent.

Consent for publication: All authors consent for publication of article in Military Medical Research journal.

Availability of data and materials: The datasets used and/or analyzed during the current study are available from the corresponding author on reasonable request.

Competing interests: The authors have no competing interests.

Funding: This study was financially supported by the AJA University of Medical Sciences.

\section{Authors' contributions:}

\section{1. hamidi farahani, Ramin}

Conceptualization

Data curation

Funding acquisition

Methodology

Project administration

Writing-original draft

\section{2. mosaed, reza}

Data curation

Investigation

Methodology

Resources

Writing-review \& editing

\section{Nezami-Asl, amir}

Formal analysis

Investigation

Methodology 
Resources

Software

Validation

Visualization

Writing-review \& editing

\section{4. chamanara, Mohsen}

Data curation

Formal analysis

Investigation

Methodology

Validation

Visualization

Writing-review \& editing

\section{Soleiman-Meigooni, saeed}

Formal analysis

Investigation

Methodology

Resources

Writing-original draft

\section{6. kalantar, Shahab}

Investigation

Methodology

Software

Validation

Visualization

Writing-review \& editing

7. Mojtaba Yousefi zoshk

Methodology

Software

Validation

Investigation

Writing-review \& editing 


\section{8. shiri malek abad, ebad}

Data curation

Investigation

Methodology

Software

Validation

Visualization

Writing-review \& editing

\section{9. hazrati, ebrahim}

Conceptualization

Investigation

Methodology

Project administration

Resources

Supervision

Validation

Writing-original draft

\section{Acknowledgments}

Not to mention.

\section{References}

1. Wang C, Horby PW, Hayden FG, Gao GF. "A novel coronavirus outbreak of global health concern," (in eng), Lancet, vol. 395, no. 10223, pp. 470-473, Feb 152020.

2. (June 14). Coronavirus Outbreak. Available: https://www.worldometers.info/coronavirus/.

3. X Yang et al., "Clinical course and outcomes of critically ill patients with SARS-CoV-2 pneumonia in Wuhan, China: a single-centered, retrospective, observational study," 2020.

4. $\mathrm{N}$ Chen et al., "Epidemiological and clinical characteristics of 99 cases of 2019 novel coronavirus pneumonia in Wuhan, China: a descriptive study" vol. 395, no. 10223, pp. 507-513, 2020.

5. Hamidi Farahani R, et al. "Clinical Features of ICU Admitted and Intubated Novel Corona Virusinfected Patients in Iran," (in en \%J. Arch Clin Infect Dis) Research Article vol. 2020;15(2):e103295.

6. T Kuiken et al., "Newly discovered coronavirus as the primary cause of severe acute respiratory syndrome" vol. 362, no. 9380, pp. 263-270, 2003.

7. Zaki AM, Van Boheemen S, Bestebroer TM, Osterhaus AD. and R. A. J. N. E. J. o. M. Fouchier, "Isolation of a novel coronavirus from a man with pneumonia in Saudi Arabia" vol. 367, no. 19, pp. 1814-1820, 2012. 
8. Z Xu et al., "Pathological findings of COVID-19 associated with acute respiratory distress syndrome" vol. 8, no. 4, pp. 420-422, 2020.

9. Fox SE, Akmatbekov A, Harbert JL, Li G, Brown JQ. and R. S. J. T. L. R. M. Vander Heide, "Pulmonary and cardiac pathology in African American patients with COVID-19: an autopsy series from New Orleans," 2020.

10. Y-H Jin et al., "A rapid advice guideline for the diagnosis and treatment of 2019 novel coronavirus (2019-nCoV) infected pneumonia (standard version)" vol. 7, no. 1, p. 4, 2020.

11. P Conti et al., "Induction of pro-inflammatory cytokines (IL-1 and IL-6) and lung inflammation by Coronavirus-19 (COVI-19 or SARS-CoV-2): anti-inflammatory strategies" vol. 34, no. 2, p. 1, 2020.

12. W Zhang et al., "The use of anti-inflammatory drugs in the treatment of people with severe coronavirus disease 2019 (COVID-19): The experience of clinical immunologists from China" p. $108393,2020$.

13. Kinoshita Y, Ishii H, Kushima H, Watanabe K. M. J. A. m. Fujita, and surgery, "High-dose steroid therapy for acute respiratory distress syndrome lacking common risk factors: predictors of outcome" vol. 5, no. 2, pp. 146-153, 2018.

14. Orens JB, Martinez FJ, Lynch JP 3rd, "Pleuropulmonary manifestations of systemic lupus erythematosus," (in eng), Rheum Dis Clin North Am, vol. 20, no. 1, pp. 159 - 93, Feb 1994.

15. Chen $M C$, Wu $Y L$, Lee $K L$, Lai KS, Chung $C L$, "Lupus pneumonitis presenting with high titre of anti-Ro antibody," (in eng), Respirol Case Rep, vol. 6, no. 1, p. e00280, Jan 2018.

16. Şişmanlar Eyüboğlu T, Aslan AT, Özdemir Y, Gezgin Yıldııım D, Buyan N, Boyunağa Ö, "Isolated acute lupus pneumonitis as the initial presentation of systemic lupus erythematosus in an 8-year-old girl," (in eng), Auto Immun Highlights, vol. 9, no. 1, p. 4, Mar 272018.

17. McGonagle D, O'Donnell JS, Sharif K, Emery P, Bridgewood C. "Immune mechanisms of pulmonary intravascular coagulopathy in COVID-19 pneumonia" The Lancet Rheumatology, 2020.

18. Coperchini F, Chiovato L, Croce L, Magri F, Rotondi MJC, Reviews GF. "The cytokine storm in COVID19: an overview of the involvement of the chemokine/chemokine-receptor system," 2020.

19. Channappanavar R, Perlman S, "Pathogenic human coronavirus infections: causes and consequences of cytokine storm and immunopathology," in Seminars in immunopathology, 2017, vol. 39, no. 5, pp. 529-539: Springer.

20. Wong JJM, Leong JY, Lee JH, Albani S. and J. G. J. A. o. t. m. Yeo, "Insights into the immunopathogenesis of acute respiratory distress syndrome" vol. 7, no. 19, 2019.

21. W-j. Guan et al., "Clinical characteristics of 2019 novel coronavirus infection in China" 2020.

22. Veronese N, et al. "Use of Corticosteroids in Coronavirus Disease 2019 Pneumonia: A Systematic Review of the Literature". Frontiers in Medicine. 2020;7:170.

23. Y Wang et al., "Early, low-dose and short-term application of corticosteroid treatment in patients with severe COVID-19 pneumonia: single-center experience from Wuhan, China," 2020. 
24. (June 19, 2020). low-cost dexamethasone reduces death by up to one third in hospitalised patients with severe respiratory complications of COVID-19. News release. University of Oxford. June 16, 2020. Available: http://www.ox.ac.uk/news/2020-06-16-low-cost-dexamethasone-reduces-death-onethird-hospitalised-patients-severe.

\section{Figures}

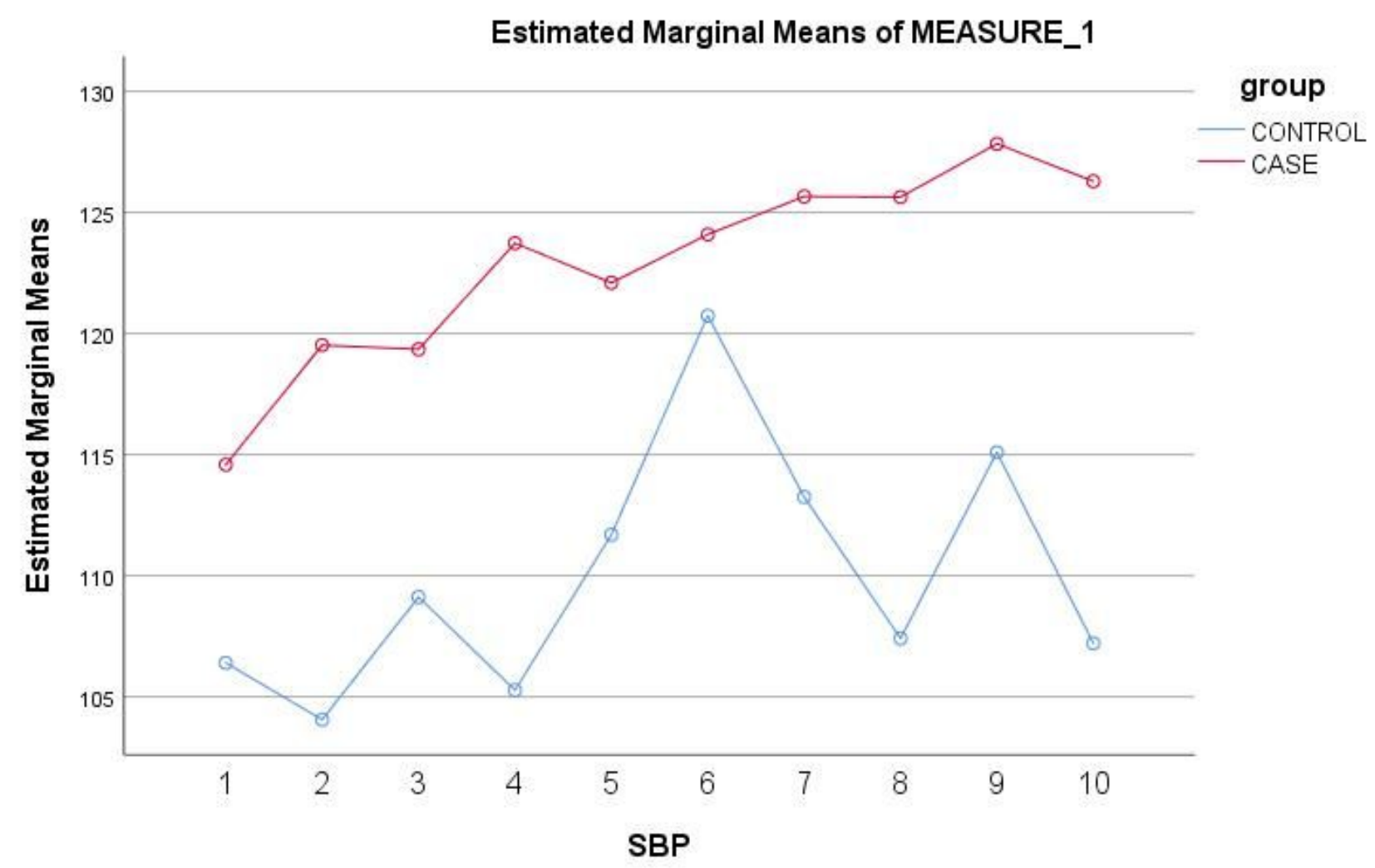

Covariates appearing in the model are evaluated at the following values: age $=64.03$

\section{Figure 1}

Means of case and control group's SBP in ten days 


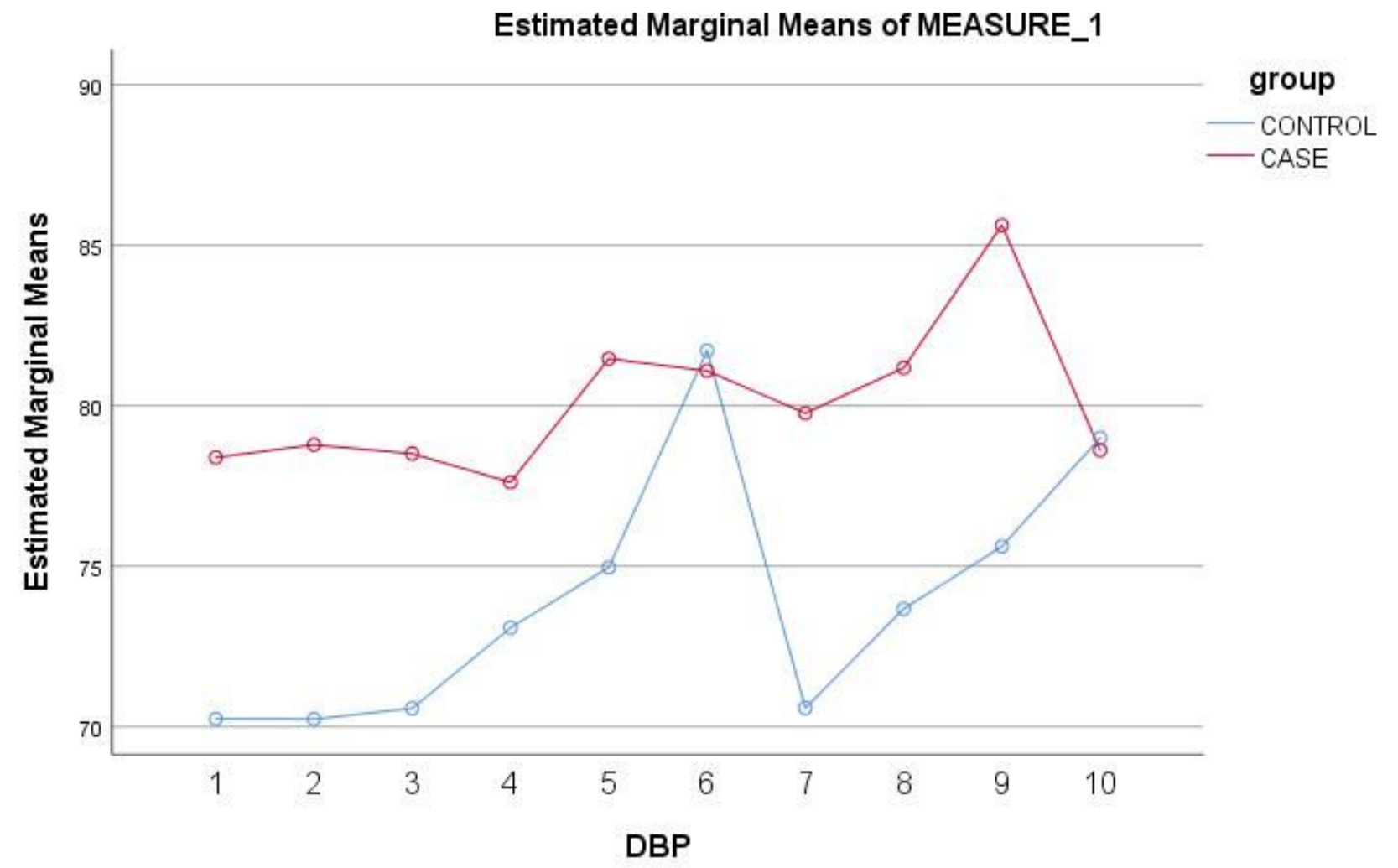

Covariates appearing in the model are evaluated at the following values: age $=64.03$

Figure 2

Means of case and control group's DBP in ten days 


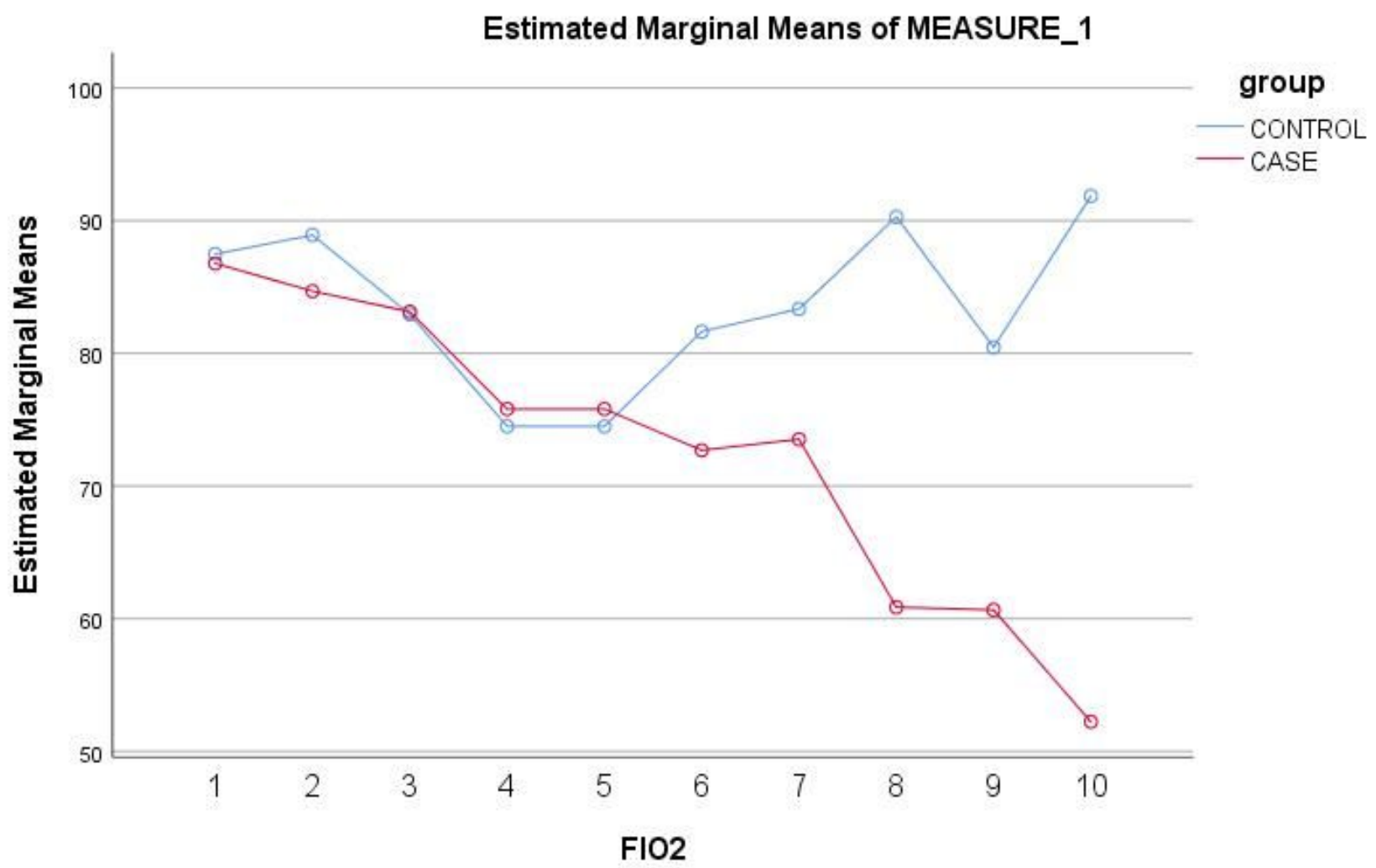

Covariates appearing in the model are evaluated at the following values: age $=63.41$

\section{Figure 3}

Means of case and control group's FiO2 in ten days 


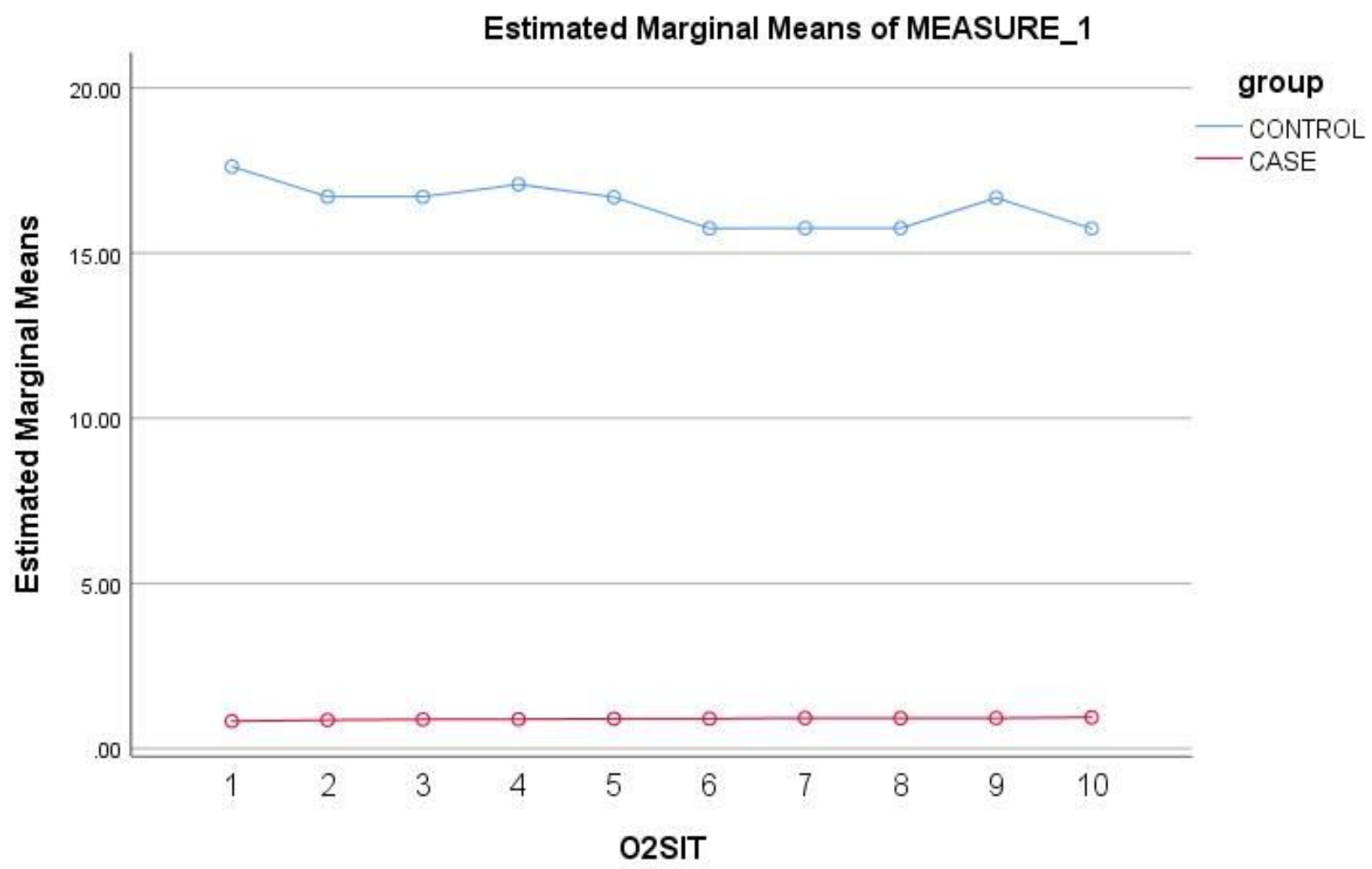

Covariates appearing in the model are evaluated at the following values: age $=64.03$

\section{Figure 4}

Means of case and control group's 02sat in ten days 


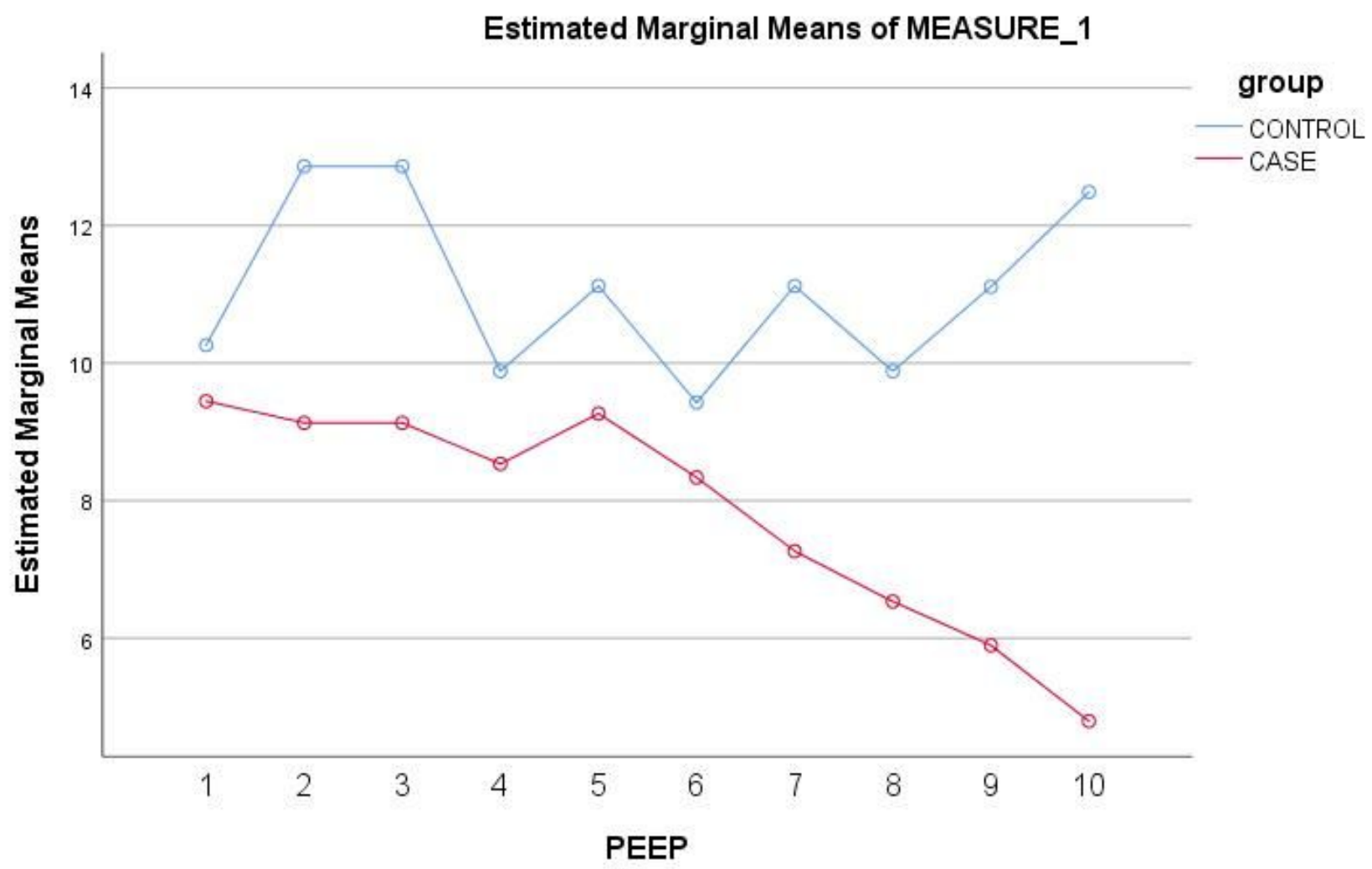

Covariates appearing in the model are evaluated at the following values: age $=62.00$

Figure 5

Means of case and control group's PEEP in ten days 


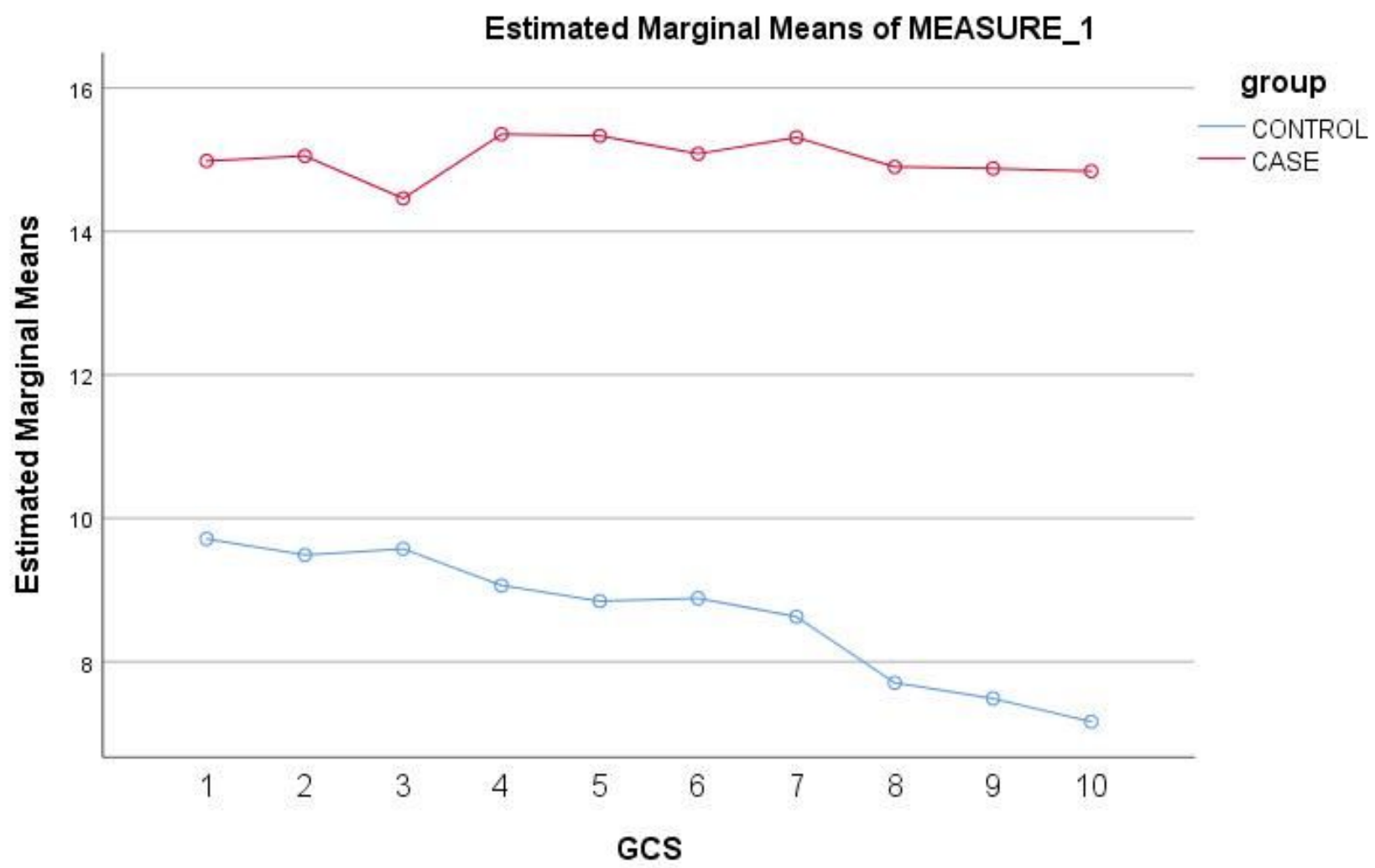

Covariates appearing in the model are evaluated at the following values: age $=64.03$

\section{Figure 6}

Means of case and control group's GCS in ten days 


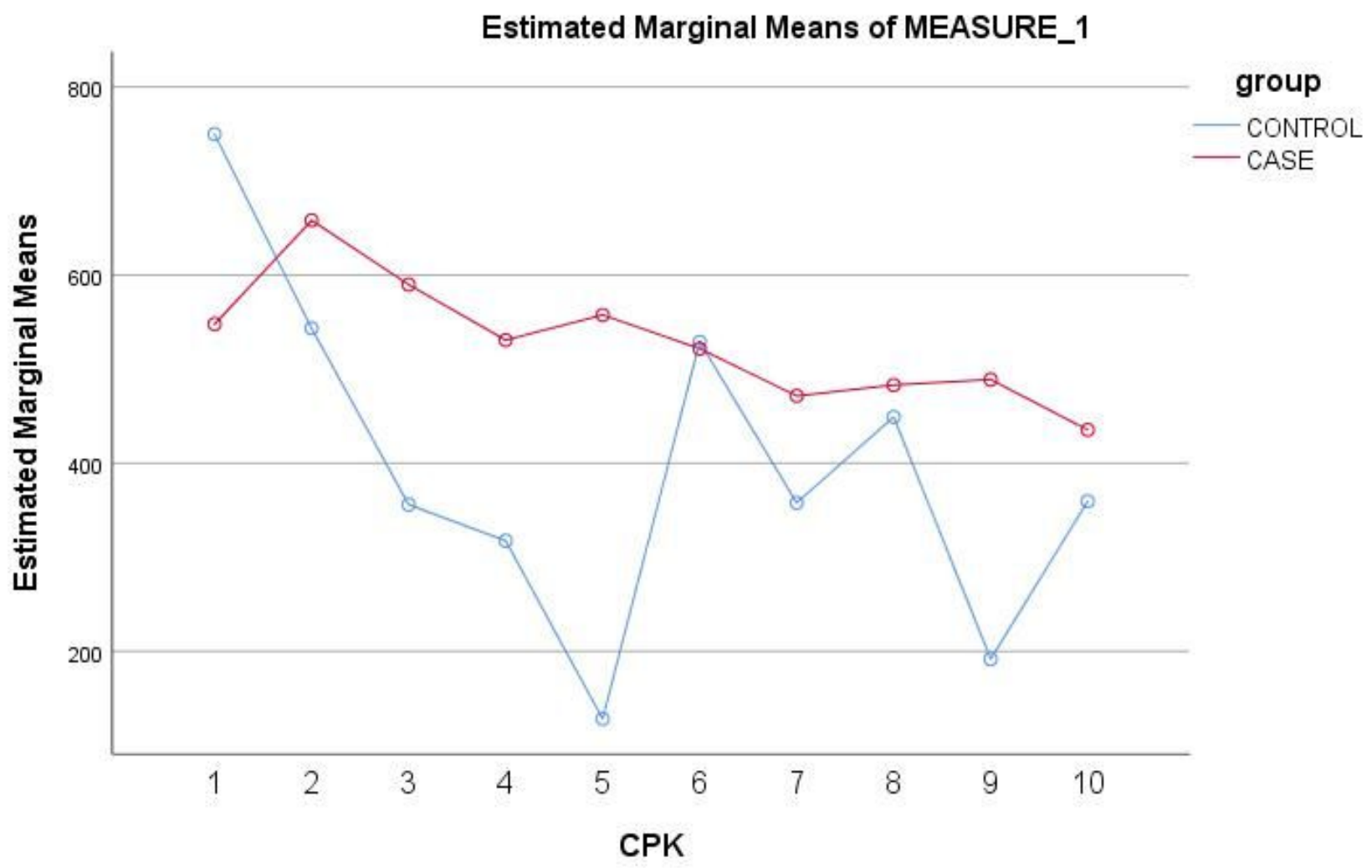

Covariates appearing in the model are evaluated at the following values: age $=57.14$

Figure 7

Means of case and control group's CPK in ten days 


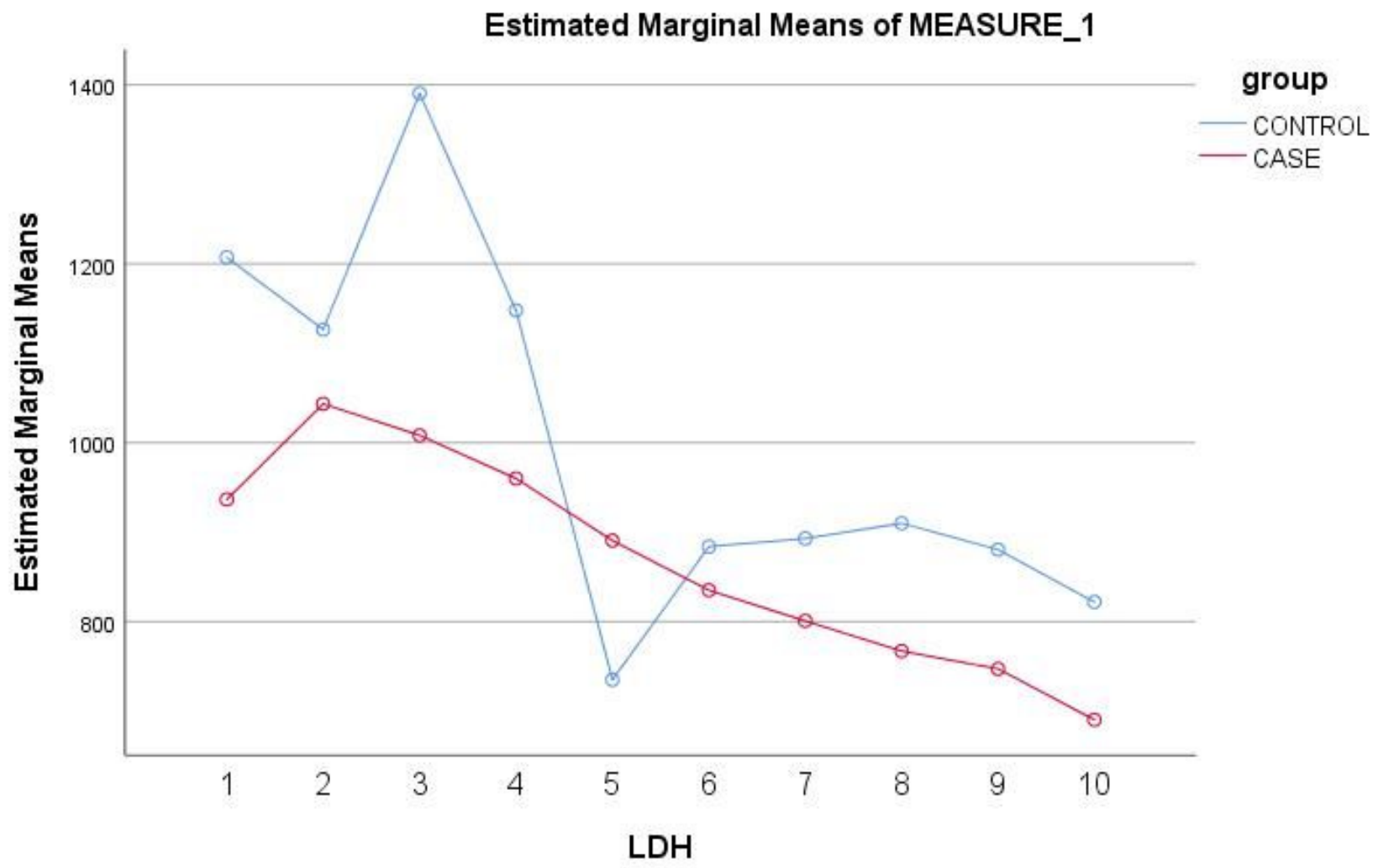

Covariates appearing in the model are evaluated at the following values: age $=55.65$

\section{Figure 8}

Means of case and control group's LDH in ten days
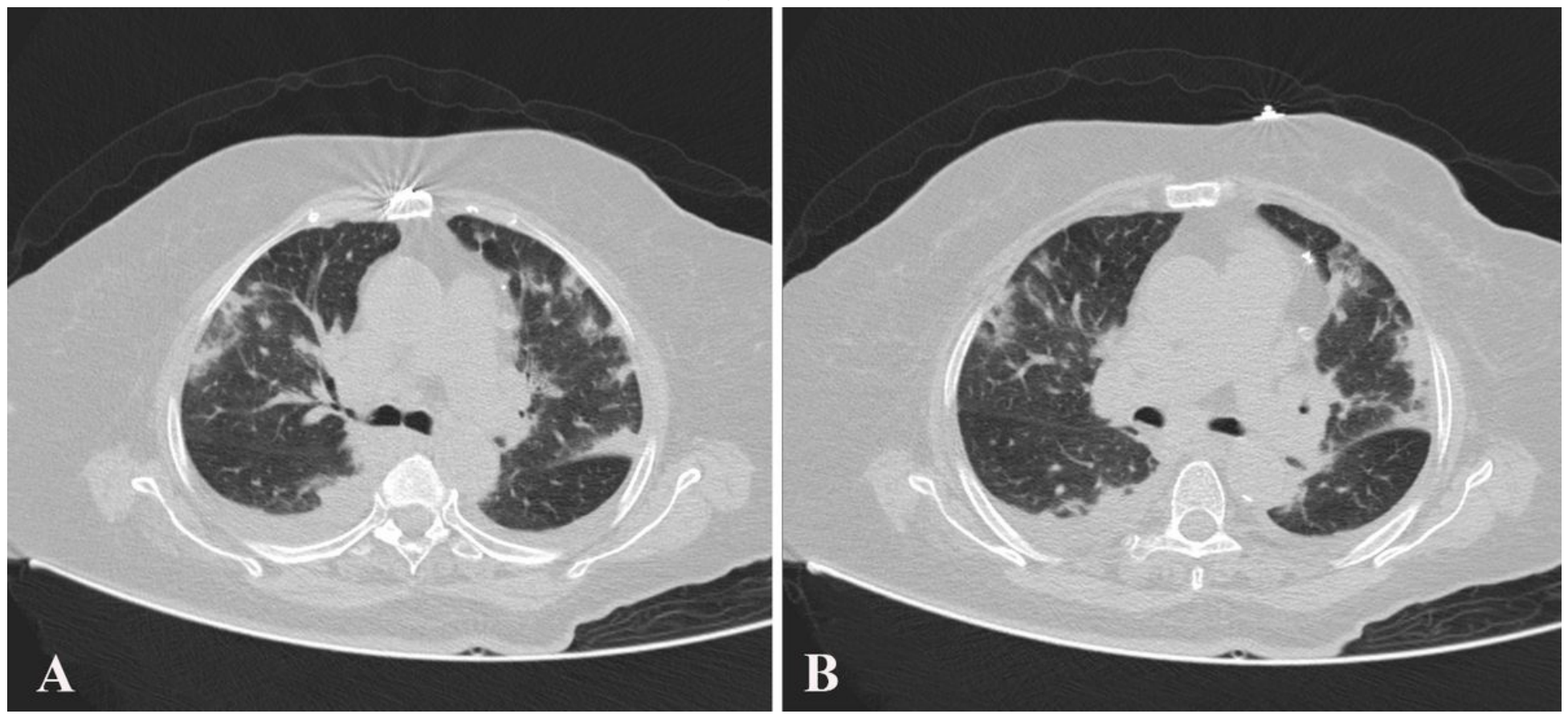

Figure 9

Pretreatment Chest CT scan 

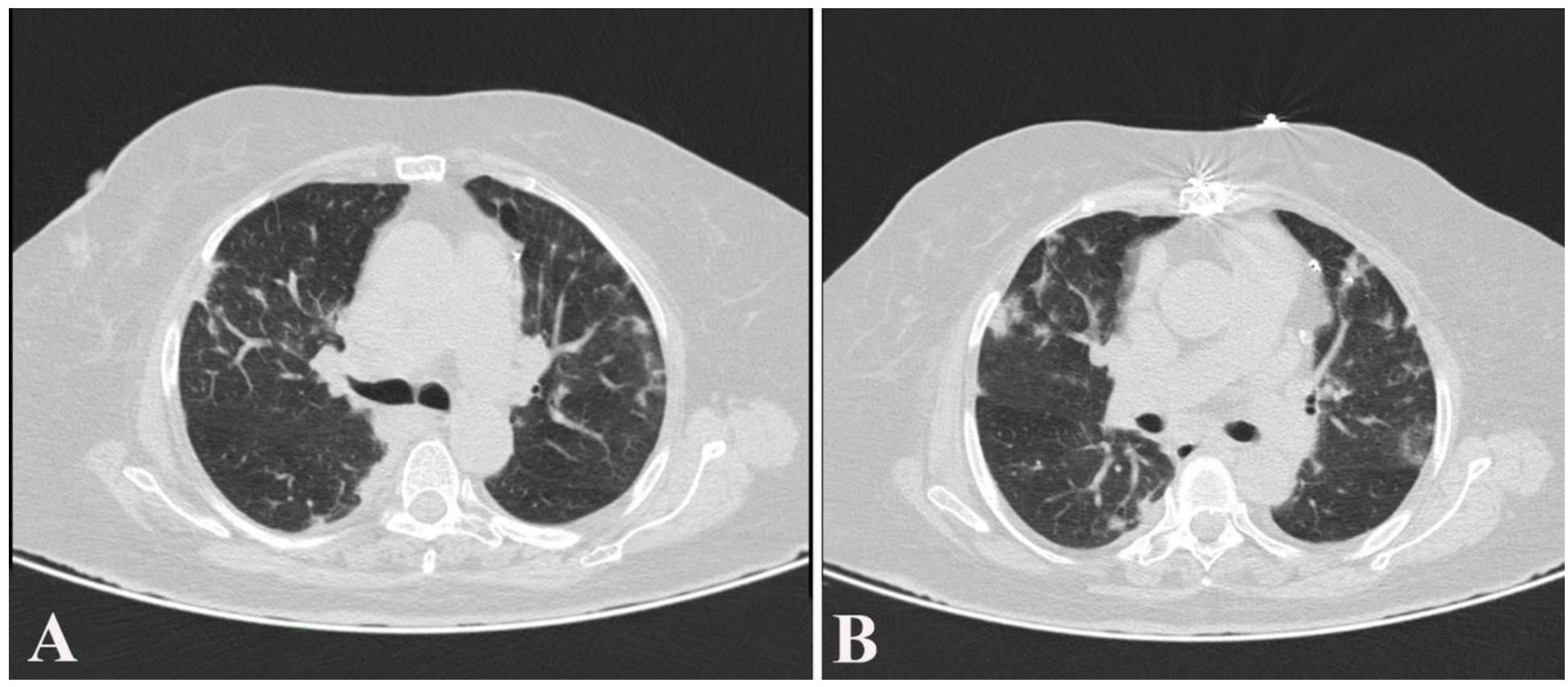

Figure 10

Post-treatment (3 days after receiving methylprednisolone) Chest CT-scan 